\title{
A Mixed Problem for Hyperbolic Equations of Second Order with a First Order Derivative Boundary Condition
}

\author{
By \\ Mitsuru IKawA*
}

\section{§1. Introduction}

On the mixed problems for hyperbolic equations of second order, only the problems with the Dirichlet condition and with the Neumann condition are studied satisfactorily. Concerning the problems with the other boundary conditions Agmon [1] contains the results on more general boundary conditions in the case when the domain is a half space and the coefficients are constant. And the author showed the not well-posedness in $L^{2}$-sense of the problem for the wave equation with an oblique derivative boundary condition [7].

In this paper we extend the results for second order equations of Agmon's paper to the case of variable coefficients and a general domain.

Let $S$ be a sufficiently smooth compact hypersurface in $R^{n}$ and let $\Omega$ be the interior or exterior domain of $S$. Consider a hyperbolic equation of second order

$$
\begin{aligned}
L=\frac{\partial^{2}}{\partial t^{2}}+a_{1}(x, t: D) \frac{\partial}{\partial t} & +a_{2}(x, t: D) \\
a_{1}(x, t: D)= & \sum_{j=1}^{n} 2 h_{j}(x, t) \frac{\partial}{\partial x_{j}}+h(x, t) \\
a_{2}(x, t: D)= & -\sum_{i, j=1}^{n} a_{i j}(x, t) \frac{\partial^{2}}{\partial x_{i} \partial x_{j}} \\
& + \text { first order }
\end{aligned}
$$

Received June 19, 1969.

Communicated by S. Matsuura.

* Department of Mathematics, Osaka University. 
where all the coefficients are in $\mathscr{B}(\Omega \times(0, T))^{1)}$ and $h_{j}(x, t)$ $(j=1,2, \cdots, n)$ are real-valued and $a_{2}(x, t: D)$ is an elliptic operator satisfying

$$
\begin{gathered}
\sum_{i, j=1}^{n} a_{i j}(x, t) \xi_{i} \xi_{j}>d \sum_{i=1}^{n} \xi_{i}^{2} \quad(d>0) \\
a_{i j}(x, t)=a_{j i}(x, t)
\end{gathered}
$$

for all $(x, t, \xi) \in \Omega \times[0, T] \times R^{n}$, and consider a boundary operator

$$
B=\frac{\partial}{\partial v_{t}}-\sigma_{1}(s, t) \frac{\partial}{\partial t}+\sigma_{2}(s, t)
$$

where $\frac{\partial}{\partial v_{t}}$ is defined by

$$
\frac{\partial}{\partial v_{t}}=\sum_{i=1}^{n} v_{i}(s, t) \frac{\partial}{\partial x_{i}},
$$

$v(s, t)=\left(v_{1}(s, t), \cdots, v_{n}(s, t)\right)$ is a smooth vector defined on $S \times[0, T]$, and $\sigma_{i}(s, t) \quad(i=1,2)$ is a sufficiently smooth function defined on $S \times[0, T]$ and $\sigma_{1}(s, t)$ is real-valued.

Our problem is to obtain a function $u(x, t) \in \mathcal{E}_{t}^{0}\left(H^{2}(\Omega)\right) \cap \mathcal{E}_{t}^{1}\left(H^{1}(\Omega)\right)$ $\cap \mathcal{E}_{t}^{2}\left(L^{2}(\Omega)\right)^{2)}$ satisfying

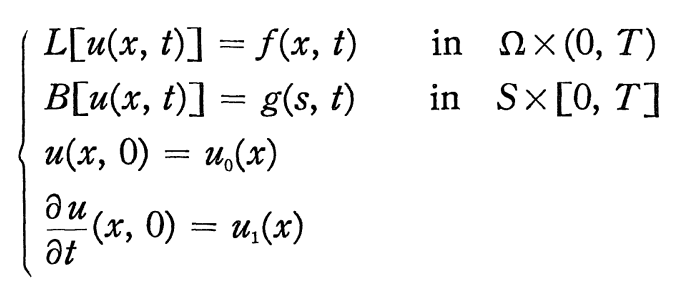

for given inital data $\left\{u_{0}(x), u_{1}(x)\right\}$, second member $f(x, t)$ and boundary data $g(s, t)$. We denote this problem by $P(L, B)$. When we want to treat this problem in $L^{2}$-sense it is necessary to pose some conditions on $v(s, t)$ and $\sigma_{1}(s, t)$ that

$$
\sigma_{1}(s, t)<\sum_{j=1}^{n} h_{j}(s, t) \nu_{j}(s)
$$

and $v(s, t)$ is not so far away from the conormal vector $n(s, t)$ $=\left(\sum_{j=1}^{n} a_{1 j} \nu_{j}, \cdots, \sum_{j=1}^{n} a_{n j} \nu_{j}\right)$ of $a_{2}(x, t: D)$ where $\nu(s)=\left(\nu_{1}(s), \cdots, \nu_{n}(s)\right)$ is

1) $\mathcal{B}(\omega), \omega$ being an open set, is the set of all $C^{\infty}$-functions defined in $\omega$ such that their all partial derivatives of any order are bounded.

2) $u(x, t) \in \mathcal{E}_{t}^{m}(E)$ means that $u(x, t)$ is $m$-times continuously differentiable in $t$ as $E$-valued function. 
the unit outer normal of $S$ at $s .^{3)}$ This condition will be prescribed precisely in $\S 3$.

In section 2 the energy inequality for $P(L, B)$ is derived and in section 3 we prove the existence of the solution by using the energy inequality and the existence theorem for the case $v(s, t)=n(s, t)$ proved in [8].

In our treatment the essential point is to derive the energy inequality for $P(L, B)$. To prove this a certain estimate of the trace of the solution plays an important role, which follows from only $L u=f$.

The author wishes to express his sincere gratitude to Professor S. Mizohata and Professor H. Tanabe for their invaluable suggestions and continuous encouragement.

\section{§2. Energy Inequality}

We note some notations which will be used. Let $\Omega$ be $R_{+}^{n}$ or a domain with a sufficiently smooth compact boundary. $\langle\cdot\rangle_{i, 0,2}$ denotes the norm of the space $H^{i}(\partial \Omega)$. For $u(x) \in H^{p+1}(\Omega)(p \geqslant 0)$, $\langle u(x)\rangle_{p, \partial \Omega}$ means the $p$-th norm of the trace of $u(x)$ to the boundary $\partial \Omega$. For $u(x, t) \in \mathcal{E}_{t}^{0}\left(H^{p}(\Omega)\right) \cap \mathcal{E}_{t}^{1}\left(H^{p-1}(\Omega)\right) \cap \cdots \cap \mathcal{E}_{t}^{p}\left(L^{2}(\Omega)\right)$ we define $\||\cdot|\|_{p, \Omega}$ by

$$
\left|\|u(x, t) \mid\|_{p, \Omega}^{2}=\sum_{i=0}^{p}\left\|\left(\frac{\partial}{\partial t}\right)^{i} u(x, t)\right\|_{p-i, L^{2}(\Omega)}^{2}\right.
$$

and for $g(s, t) \in \mathcal{E}_{t}^{0}\left(H^{p}(\partial \Omega)\right) \cap \mathcal{E}_{t}^{1}\left(H^{p-1}(\partial \Omega)\right) \cap \cdots \cap \mathcal{E}_{t}^{p}\left(L^{2}(\partial \Omega)\right) \ll \cdot \gg_{p, \partial \Omega}$ by

$$
\ll g(s, t)\rangle_{p, \partial \Omega}^{2}=\sum_{i=0}^{n}\left\langle\left(\frac{\partial}{\partial t}\right)^{i} g(s, t)\right\rangle_{p-i, \partial \Omega}^{2} .
$$

It is obvious that

$$
\left(\sum_{i, j=1}^{n}\left(a_{i j}(x, t) \frac{\partial u}{\partial x_{i}}, \frac{\partial u}{\partial x_{j}}\right)+(u, u)\right)^{1 / 2}
$$

gives an equivalent norm in $H^{1}(\Omega)$ from the condition (1.2). Then if we put

3) See Theorem 1 of [9]. 
(2.3) $\quad\|u(x, t)\|_{\mathscr{H}(t)}=\left(\sum_{i, j=1}^{n}\left(a_{i j} \frac{\partial u}{\partial x_{i}}, \frac{\partial u}{\partial x_{j}}\right)+(u, u)+\left(\frac{\partial u}{\partial t}, \frac{\partial u}{\partial t}\right)\right)^{1 / 2}$,

for $u(x, t) \in \mathcal{E}_{t}^{0}\left(H^{1}(\Omega)\right) \cap \mathcal{E}_{t}^{1}\left(L^{2}(\Omega)\right)$, there exists a constant $M$ such that

$$
\frac{1}{M}\|u(x, t)\|_{\mathcal{H}(t)} \leqslant\|u(x, t)\|\left\|_{1, Q} \leqslant M\right\| u(x, t) \|_{\mathcal{H}(t)} .
$$

Concerning the vector $v(s, t)$ which is not tangential to $S$ we can assume without loss of generality that

$$
\sum_{i, j=1}^{n} a_{i j}(s, t) \nu_{i}(s) \nu_{j}(s)=\sum_{i=1}^{n} v_{i}(s, t) \nu_{i}(s)
$$

holds on $S \times[0, T]$. Let us set

$$
b(s, t)^{2}=\sum_{i=1}^{n}\left|\sum_{j=1}^{n} a_{i j}(s, t) \nu_{j}(s)-v_{i}(s, t)\right|^{2}
$$

and

$$
b_{0}=\sup _{s \times[0, T]} b(s, t) .
$$

Since $B$ satisfies the condition (1.5)

$$
\alpha(s, t)=\sum_{i=1}^{n} h_{i}(s, t) \nu_{i}(s)-\sigma_{1}(s, t)
$$

is a positive smooth function, and set

$$
\alpha_{0}=\inf _{S \times[0, T]} \alpha(s, t)
$$

We state a simple lemma without proof.

Lemma 2.1. Let $\gamma(t)$ and $\rho(t)$ be positive, and defined on $(0, a)$ $(a>0)$. If $\gamma(t)$ is summable on $(0, a)$ and $\rho(t)$ is increasing and

$$
\gamma(t) \leqslant c \int_{0}^{t} \gamma(s) d s+\rho(t)
$$

holds, then we have

$$
\gamma(t) \leqslant e^{c t} \rho(t)
$$

Energy inequality in the case $\Omega=\boldsymbol{R}_{+}^{n}$. We should like to consider at first the case when the domain is a half space $R_{+}^{n}=\left\{\left(x^{\prime}, x_{n}\right)\right.$; $\left.x^{\prime} \in R^{n-1}, x_{n}>0\right\}$. In this paragraph we omit for the simplicity the 
notation $\Omega$ in (2.1) and $\partial \Omega$ in (2.2). Let $N$ be the first order differential operator defined by

$$
N=-\sum_{j=1}^{n} a_{n j}(x, t) \frac{\partial}{\partial x_{j}}+h_{n}(x, t) \frac{\partial}{\partial t} .
$$

Lemma 2. 2. There exists constants $C_{1}, C_{2}, C_{3}$ determined by $L$ such that for any function $u(x, t) \in \mathcal{E}_{t}^{0}\left(H^{2}\left(R_{+}^{n}\right)\right) \cap \mathcal{E}_{t}^{1}\left(H^{1}\left(R_{+}^{n}\right)\right) \cap \mathcal{E}_{t}^{2}\left(L^{2}\left(R_{+}^{n}\right)\right)$ the following estimate holds:

$$
\begin{aligned}
\frac{d^{2}}{2} \int_{0}^{t}\langle u(x, t)\rangle_{1}^{2} d t \leqslant \int_{0}^{t}\langle(N u)(x, t)\rangle_{0}^{2} d t+C_{1} \int_{0}^{t}\left\langle\frac{\partial u}{\partial t}(x, t)\right\rangle_{0}^{2} d t \\
+C_{2}\left(\|u(x, t)\|_{\mathscr{H}(t)}^{2}+\|u(x, 0)\|_{\mathscr{H}(0)}^{2}\right) \\
+C_{3} \int_{0}^{t}\|u(x, t)\|_{\mathscr{H}(t)}^{2} d t+\int_{0}^{t}\|L[u(x, t)]\|_{L^{2}\left(R_{+}^{n}\right)}^{2} d t
\end{aligned}
$$

Proof. We get by integration by parts for some constant $C_{3}^{\prime}$

$$
\begin{aligned}
& \int_{0}^{t} d t \int_{R_{+}^{n}}\left(L u \overline{N u}+N u \overline{L u}\left(x^{\prime}, x_{n}, t\right) d x^{\prime} d x_{n}\right. \\
& \geqslant \int_{0}^{t} d t \int_{R^{n-1}} d x^{\prime}\left(-a_{n n}\left|\frac{\partial u}{\partial t}\right|^{2}+4 \operatorname{Re} \sum_{j=1}^{n-1} a_{n j} \frac{\partial u}{\partial x_{j}} h_{n} \frac{\partial u}{\partial t}\right. \\
& \quad-2 \operatorname{Re} \sum_{i=1}^{n-1} h_{i} \frac{\partial u}{\partial x_{i}} a_{n n} \frac{\overline{\partial u}}{\partial t}-2 h_{n}^{2}\left|\frac{\partial u}{\partial t}\right|^{2} \\
& +\sum_{i, j=1}^{n-1} a_{n n} a_{i j} \frac{\partial u}{\partial x_{i}} \frac{\partial u}{\partial x_{j}}-2\left|\sum_{j=1}^{n-1} a_{n j} \frac{\partial u}{\partial x_{j}}\right|^{2} \\
& \quad-2 \operatorname{Re} a_{n n} \frac{\partial u}{\partial x_{n}} \sum_{j=1}^{n-1} a_{n j} \frac{\partial u}{\partial x_{j}} \\
& \left.\quad-a_{n n}^{2}\left|\frac{\partial u}{\partial x_{n}}\right|^{2}+2 \operatorname{Re} a_{n n} h_{n} \frac{\partial u}{\partial x_{n}} \frac{\partial u}{\partial t}\right)\left(x^{\prime}, 0, t\right) \\
& +\int_{R_{+}^{n}} d x^{\prime} d x_{n}\left[\left(-2 \operatorname{Re} \frac{\partial u}{\partial t} \sum_{j=1}^{n-1} a_{n j} \frac{\overline{\partial u}}{\partial x_{j}}-2 \operatorname{Re} \frac{\partial u}{\partial t} a_{n n} \frac{\partial u}{\partial x_{n}}\right.\right. \\
& +h_{n}\left|\frac{\partial u}{\partial t}\right|^{2}-2 \operatorname{Re} \sum_{i, j=1}^{n-1} h_{i} a_{n j} \frac{\partial u}{\partial x_{i}} \frac{\overline{\partial u}}{\partial x_{j}} \\
& \left.\left.\quad-2 \operatorname{Re} \sum_{i=1}^{n-1} a_{n n} \frac{\partial u}{\partial x_{n}} h_{i} \frac{\partial u}{\partial x_{i}}-h_{n} a_{n n}\left|\frac{\partial u}{\partial x_{n}}\right|^{2}\right)\left(x^{\prime}, x_{n}, t\right)\right]_{0}^{t} \\
& \quad-C_{3} \int_{0}^{t}\|u(x, t)\|_{\mathcal{H}(t)}^{2} d t \\
& =I+I I+I I I .
\end{aligned}
$$


The integrand of $I$ equals

$$
\begin{aligned}
& a_{n n} \sum_{i, j=1}^{n-1} a_{i j} \frac{\partial u}{\partial x_{i}} \frac{\overline{\partial u}}{\partial x_{j}}-\left|\sum_{j=1}^{n-1} a_{n j} \frac{\partial u}{\partial x_{j}}\right|^{2}-|N u|^{2} \\
&-\left(a_{n n}+h_{n}^{2}\right)\left|\frac{\partial u}{\partial t}\right|^{2}+2 \operatorname{Re} \sum_{j=1}^{n-1} a_{n j} \frac{\partial u}{\partial x_{j}} h_{n} \frac{\partial u}{\partial t} \\
&-2 \operatorname{Re} \sum_{j=1}^{n-1} h_{j} \frac{\partial u}{\partial x_{j}} a_{n n} \frac{\overline{\partial u}}{\partial t},
\end{aligned}
$$

since it follows from (1.2) that

$$
\begin{gathered}
a_{n n} \sum_{i, j=1}^{n-1} a_{i j} \xi_{i} \xi_{j}-\left|\sum_{j=1}^{n-1} a_{n j} \xi_{j}\right|^{2} \geqslant d^{2} \sum_{j=1}^{n-1} \xi_{j}^{2} \\
\geqslant d^{2} \sum_{j=1}^{n-1}\left|\frac{\partial u}{\partial x_{j}}\right|^{2}-|N u|^{2}-\left(a_{n n}+h_{n}^{2}\right)\left|\frac{\partial u}{\partial t}\right|^{2} \cdot \\
\quad-\frac{1}{q}\left(\sum_{j=1}^{n-1}\left(a_{n j}^{2}+h_{j}^{2}\right)\right) \sum_{j=1}^{n-1}\left|\frac{\partial u}{\partial x_{j}}\right|^{2}-q\left(h_{n}^{2}+a_{n n}^{2}\right)\left|\frac{\partial u}{\partial t}\right|^{2} .
\end{gathered}
$$

We have by taking $q=2 \sum_{j=1}^{n-1}\left(a_{n j}^{2}+h_{j}^{2}\right) / d^{2}$

$$
\begin{aligned}
I \geqslant & \frac{d^{2}}{2} \int_{0}^{t} \sum_{j=1}^{n-1}\left\langle\frac{\partial u}{\partial x_{j}}\right\rangle^{2} d t-\int_{0}^{t}\langle N u\rangle^{2} d t \\
& \quad-\int_{0}^{t} d t \int_{R^{n-1}} d x^{\prime}\left(a_{n n}+h_{n}^{2}+2\left(a_{n n}^{2}+h_{n}^{2}\right) \frac{\sum_{j=1}^{n-1}\left(a_{n j}^{2}+h_{j}^{2}\right)}{d^{2}}\left|\frac{\partial u}{\partial t}\right|^{2}\right) .
\end{aligned}
$$

Put

$$
\text { (2.11) } C_{1}=\sup _{\left(x^{\prime}, t\right) \in \boldsymbol{R}^{n-1} \times[0, T]}\left(a_{n n}+h_{n}^{2}+2\left(h_{n}^{2}+a_{n n}^{2}\right) \frac{\sum_{j=1}^{n-1}\left(a_{n j}^{2}+h_{j}^{2}\right)}{d^{2}}\right)\left(x^{\prime}, 0, t\right)
$$

and it follows

$$
I \geqslant \frac{d^{2}}{2} \int_{0}^{t} \sum_{j=1}^{n-1}\left\langle\frac{\partial u}{\partial x_{j}}\right\rangle^{2} d t-\int_{0}^{t}\langle N u\rangle^{2} d t-C_{1} \int_{0}^{t}\left\langle\frac{\partial u}{\partial t}\right\rangle^{2} d t .
$$

The absolute value of the integrand of $I I$ is majorated by

$$
\begin{aligned}
& 2\left|\sum_{j=1}^{n-1} a_{n j} \frac{\partial u}{\partial x_{j}}\right|^{2}+2\left|\frac{\partial u}{\partial t}\right|^{2}+2 a_{n n}^{2}\left|\frac{\partial u}{\partial x_{n}}\right|^{2}+\left|h_{n}\right|\left|\frac{\partial u}{\partial t}\right|^{2} \\
& \quad+2\left|\sum_{j=1}^{n-1} h_{j} \frac{\partial u}{\partial x_{j}}\right|^{2}+\left|h_{n}\right| a_{n n}\left|\frac{\partial u}{\partial x_{n}}\right|^{2}+\left|h_{n}\right| \sum_{i, j=1}^{n-1} a_{i j} \frac{\partial u}{\partial x_{i}} \overline{\frac{\partial u}{\partial x_{j}}} \\
& \quad \geqslant 2\left(\sum_{i, j=1}^{n} a_{i j}^{2}+\sum_{j=1}^{n} h_{j}^{2}\right) \sum_{j=1}^{n}\left|\frac{\partial u}{\partial x_{j}}\right|^{2}+\left(\left|h_{n}\right|+2\right)\left|\frac{\partial u}{\partial t}\right|^{2} .
\end{aligned}
$$


Put

(2. 12)

$$
C_{2}=\sup _{\boldsymbol{R}_{+}^{n} \times[0, T]}\left(2\left(\sum_{i, j=1}^{n} a_{i j}^{2}+\sum_{j=1}^{n} h_{j}^{2}\right) / d+\left|h_{n}\right|+2\right)
$$

and we have

$$
|I I| \leqslant C_{2}\left(\|u(x, t)\|_{\mathscr{H}(t)}^{2}+\|u(x, 0)\|_{\mathscr{H}(0)}^{2}\right) .
$$

Then

$$
\begin{aligned}
& \int_{0}^{t}\left(\|L u\|^{2}+\|N u\|^{2}\right) d t \\
& \geqslant \frac{d^{2}}{2} \int_{0}^{t} \sum_{j=1}^{n-1}\left\langle\frac{\partial u}{\partial x_{j}}\right\rangle^{2} d t-\int_{0}^{t}\langle N u\rangle^{2} d t-C_{1} \int_{0}^{t}\left\langle\frac{\partial u}{\partial t}\right\rangle^{2} d t \\
& -C_{2}\left(\|u(t)\|_{\mathcal{H}(t)}^{2}+\|u(0)\|_{\mathcal{H}_{(0)}}^{2}\right)-C_{3}^{\prime} \int_{0}^{t}\|u(s)\|_{\mathcal{H}(s)}^{2} d s .
\end{aligned}
$$

Since $N$ is a first order operator it holds

$$
\int_{0}^{t}\|N u\|^{2} d t \leqslant \mathrm{const} . \int_{0}^{t}\|u(s)\|_{\mathscr{H}(s)}^{2} d s,
$$

thus we get (2.10).

Q.E.D.

Lemma 2. 3. There exists a constant $\widetilde{C}_{3}$ determined by $L$ such that for any solution $u(x, t) \in \mathcal{E}_{t}^{0}\left(H^{2}\left(R_{+}^{n}\right)\right) \cap \mathcal{E}_{t}^{1}\left(H^{1}\left(R_{+}^{n}\right)\right) \cap \mathcal{E}_{t}^{2}\left(L^{2}\left(R_{+}^{n}\right)\right)$ of $P(L, B)$, if

$$
b_{0} \leqslant \frac{d}{4}
$$

the estimate

$$
\begin{aligned}
\frac{d^{2}}{4} \int_{0}^{t}\langle u(x, t)\rangle_{1}^{2} d t \leqslant C_{2}\left(\|u(x, t)\|_{\mathscr{H}(t)}^{2}+\|u(x, 0)\|_{\mathscr{H}(0)}^{2}\right) \\
+\widetilde{C}_{3} \int_{0}^{t}\|u(x, t)\|_{\mathscr{H}(t)}^{2} d t+\int_{0}^{t}\|f(x, t)\|^{2} d t \\
\quad+4 \int_{0}^{t}\langle g(s, t)\rangle^{2} d t+\left(C_{1}+4 \beta^{2}\right) \int_{0}^{t}\left\langle\frac{\partial u}{\partial t}(x, t)\right\rangle^{2} d t
\end{aligned}
$$

holds for all $t \in[0, T]$, where

$$
\beta=\sup _{\left(x^{\prime}, t\right) \in \boldsymbol{R}^{n-1} \times[0, T]}\left(-h_{n}-\sigma_{1}\right) .
$$

Proof. From the boundary condition $B u\left(x^{\prime}, 0, t\right)=g\left(x^{\prime}, t\right)$ it follows 


$$
\begin{aligned}
(N u)\left(x^{\prime}, 0, t\right)= & g\left(x^{\prime}, t\right)-\sum_{j=1}^{n-1}\left(v_{j}-n_{j}\right) \frac{\partial u}{\partial x_{j}}\left(x^{\prime}, 0, t\right) \\
& -\left(-h_{n}\left(x^{\prime}, 0, t\right)-\sigma_{1}\left(x^{\prime}, t\right)\right) \frac{\partial u}{\partial t}\left(x^{\prime}, 0, t\right) \\
& -\sigma_{2}\left(x^{\prime}, t\right) u\left(x^{\prime}, 0, t\right),
\end{aligned}
$$

therefore

$$
\begin{aligned}
\langle N u\rangle^{2} \leqslant 4\left\{\langle g\rangle^{2}\right. & +b_{0}^{2}\langle u(x, t)\rangle_{1}^{2}+\beta^{2}\left\langle\frac{\partial u}{\partial t}(x, t)\right\rangle^{2} \\
& \left.+\sup _{S \times[0, T]}\left|\sigma_{2}\right|^{2}\langle u(x, t)\rangle^{2}\right\} .
\end{aligned}
$$

By inserting this estimate into (2.10)

$$
\begin{aligned}
\frac{d^{2}}{2} \int_{0}^{t}\langle u & (x, t)\rangle_{1}^{2} d t \leqslant 4 \int_{0}^{t}\left(\left\langle g\left(x^{\prime}, t\right)\right\rangle^{2}+b_{0}^{2}\langle u(x, t)\rangle_{1}^{2}\right. \\
& \left.+\beta^{2}\left\langle\frac{\partial u}{\partial t}(x, t)\right\rangle^{2}+\sup \left|\sigma_{2}\right|^{2}\langle u(x, t)\rangle^{2}\right) d t \\
& +C_{1} \int_{0}^{t}\left\langle\frac{\partial u}{\partial t}(x, t)\right\rangle^{2} d t+C_{3} \int_{0}^{t}\|u(x, t)\|_{\mathscr{H}(t)}^{2} d t \\
& +\int_{0}^{t}\|f(x, t)\|^{2} d t+C_{2}\left(\|u(x, t)\|_{\mathscr{H}(t)}^{2}+\|u(x, 0)\|_{\mathcal{H}(0)}^{2}\right)
\end{aligned}
$$

from which (2.14) follows immediately by taking account of the condition (2.13).

Q.E.D.

Proposition 2.1. Suppose that the boundary operator $B$ satisfies (1. 6) and

$$
b_{0}<\frac{d}{4} \min \left(1, \sqrt{\frac{\alpha_{0}}{C_{2}}}, \frac{\alpha_{0}}{\sqrt{C_{1}+4 \beta^{2}}}\right) .
$$

Then for any solution $u(x, t) \in \mathcal{E}_{t}^{0}\left(H^{2}\left(R_{+}^{n}\right)\right) \cap \mathcal{E}_{t}^{1}\left(H^{1}\left(R_{+}^{n}\right)\right) \cap \mathcal{E}_{t}^{2}\left(L^{2}\left(R_{+}^{n}\right)\right)$ of $P(L, B)$ the following energy inequality

$$
\begin{aligned}
& \|u(x, t)\|_{\mathscr{H}(t)}^{2}+\int_{0}^{t} \ll u(x, t) \gg_{1}^{2} d t \\
& \leqslant C\left\{\|u(x, 0)\|_{\mathscr{H}(0)}^{2}+\int_{0}^{t}\left(\|f(x, t)\|^{2}+\left\langle g\left(x^{\prime}, t\right)\right\rangle^{2}\right) d t\right\}
\end{aligned}
$$

holds for all $t \in[0, T]$, where $C$ is a constant determined by $L, B$ and $T$.

Proof. The differential inequality 


$$
\begin{aligned}
\frac{d}{d t}\|u(x, t)\|_{\mathcal{H}(t)}^{2} & \leqslant c\|u(x, t)\|_{\mathcal{H}(t)}^{2}+\|f(x, t)\|^{2} \\
& +2 \operatorname{Re} \int_{R^{n-1}}(N u)\left(x^{\prime}, 0, t\right) \overline{\frac{\partial u}{\partial t}\left(x^{\prime}, 0, t\right)} d x^{\prime}
\end{aligned}
$$

follows from $L u=f .^{4}$ ) The integration of this inequality from 0 to $t$ gives

$$
\begin{aligned}
\|u(x, t)\|_{\mathcal{H}(t)}^{2} \leqslant & \|u(x, 0)\|_{\mathcal{H}(0)}^{2}+c \int_{0}^{t}\|u(x, t)\|_{\mathscr{H}(t)}^{2} d t \\
& +\int_{0}^{t}\|f(x, t)\|^{2} d t+2 \operatorname{Re} \int_{0}^{t} d t \int_{R^{n-1}} N u \frac{\overline{\partial u}}{\partial t} d x^{\prime} .
\end{aligned}
$$

It is essential to estimate the last term. If we use oncemore the relation $(2.15)$

$$
\begin{aligned}
& 2 \operatorname{Re} \int_{0}^{t} d t \int_{R^{n-1}} N u \frac{\overline{\partial u}}{\partial t} d x^{\prime} \\
= & \int_{0}^{t} d t \int_{R^{n-1}} 2 \operatorname{Re}\left\{\left(-\frac{\partial}{\partial v_{t}}+\frac{\partial}{\partial n_{t}}\right) u \frac{\partial \bar{u}}{\partial t}-\left(-h_{n}-\sigma_{1}\right)\left|\frac{\partial u}{\partial t}\right|^{2}\right. \\
& \left.-\sigma_{2} u \frac{\overline{\partial u}}{\partial t}+g \frac{\overline{\partial u}}{\partial t}\right\} d x^{\prime}
\end{aligned}
$$

by using $\left\langle\frac{\partial u}{\partial v_{t}}-\frac{\partial u}{\partial n_{t}}\right\rangle \leqslant b_{0}\langle u\rangle_{1}$ and (2.7) (2.8)

$$
\begin{aligned}
\leqslant & \int_{0}^{t} b_{0}\langle u(x, t)\rangle_{1}\left\langle\frac{\partial u}{\partial t}\right\rangle d t-2 \alpha_{0} \int_{0}^{t}\left\langle\frac{\partial u}{\partial t}\right\rangle^{2} d t \\
& +2 \int_{0}^{t}\left\langle\sigma_{2} u\right\rangle\left\langle\frac{\partial u}{\partial t}\right\rangle d t+2 \int_{0}^{t}\langle g\rangle\left\langle\frac{\partial u}{\partial t}\right\rangle d t \\
\leqslant & -\frac{5 \alpha_{0}}{4} \int_{0}^{t}\left\langle\frac{\partial u}{\partial t}\right\rangle^{2} d t+\frac{4 b_{0}^{2}}{\alpha_{0}} \int_{0}^{t}\langle u(x, t)\rangle_{1}^{2} d t \\
& +\frac{4 \sup \left|\sigma_{2}\right|}{\alpha_{0}} \int_{0}^{t}\langle u\rangle^{2} d t+\frac{4}{\alpha_{0}} \int_{0}^{t}\langle g\rangle^{2} d t .
\end{aligned}
$$

Then it follows from $(2.18)$

$$
\begin{gathered}
\|u(x, t)\|_{\mathscr{H}(t)}^{2}+\varepsilon_{0} \int_{0}^{t} \ll u(x, t) \gg{ }_{1}^{2} d t \\
\leqslant\|u(x, 0)\|_{\mathscr{H}(0)}^{2}+\int_{0}^{t}\|f\|^{2} d t+c \int_{0}^{t}\|u(x, t)\|_{\mathcal{H}(t)}^{2} d t
\end{gathered}
$$

4) See, for example, the proof of Lemma 2.2 of [8]. 


$$
\begin{aligned}
& -\left(\frac{5 \alpha_{0}}{4}-\varepsilon_{0}\right) \int_{0}^{t}\left\langle\frac{\partial u}{\partial t}\right\rangle^{2} d t+\frac{4}{\alpha_{0}} \int_{0}^{t}\langle g\rangle^{2} d t+\text { const. } \int_{0}^{t}\langle u\rangle^{2} d t \\
& +\left(\frac{4 b_{0}^{2}}{\alpha_{0}}+\varepsilon_{0}\right) \int_{0}^{t}\langle u(x, t)\rangle_{1}^{2} d t
\end{aligned}
$$

inserting the estimate (2.14)

$$
\begin{aligned}
\leqslant & \|u(x, 0)\|_{\mathscr{H}(0)}^{2}+\int_{0}^{t}\left(\|f\|^{2}+\frac{4}{\alpha_{0}}\langle g\rangle^{2}\right) d t+c^{\prime} \int_{0}^{t}\|u(x, t)\|_{\mathscr{H}(t)}^{2} d t \\
& -\left(\frac{5 \alpha_{0}}{4}-\varepsilon_{0}\right) \int_{0}^{t}\left\langle\frac{\partial u}{\partial t}\right\rangle^{2} d t+\left(\frac{4 b_{0}^{2}}{\alpha_{0}}+\varepsilon_{0}\right) \frac{4}{d^{2}}\left\{\widetilde{C}_{3} \int_{0}^{t}\|u(x, t)\|_{\mathscr{H}(t)}^{2} d t\right. \\
& +C_{2}\left(\|u(x, t)\|_{\mathscr{H}(t)}^{2}+\|u(x, 0)\|_{\mathscr{H}(0)}^{2}\right)+\int_{0}^{t}\|f(x, t)\|^{2} d t \\
& \left.+4 \int_{0}^{t}\langle g\rangle^{2} d t+\left(C_{1}+4 \beta^{2}\right) \int_{0}^{t}\left\langle\frac{\partial u}{\partial t}\right\rangle^{2} d t\right\} .
\end{aligned}
$$

When the inequalities

$$
\begin{aligned}
& \frac{4}{d^{2}}\left(\frac{4 b_{0}^{2}}{\alpha_{0}}+\varepsilon_{0}\right) C_{2}<1-\varepsilon_{0} \\
& \frac{4}{d^{2}}\left(\frac{4 b_{0}^{2}}{\alpha_{0}}+\varepsilon_{0}\right)\left(C_{1}+4 \beta^{2}\right)<\alpha_{0}-\varepsilon_{0}
\end{aligned}
$$

hold for some $\varepsilon_{0}>0$, namely

$$
b_{0}<\frac{d}{4} \min \left(\sqrt{\frac{\alpha_{0}}{C_{2}}}, \frac{\alpha_{0}}{\sqrt{C_{1}+4 \beta^{2}}}\right),
$$

by choosing a suitable constant $C^{\prime}$ we get

$$
\begin{aligned}
& \|u(x, t)\|_{\mathcal{H}(t)}^{2}+\int_{0}^{t} \ll u(x, t) \gg_{1}^{2} d t \\
& \leqslant C^{\prime}\left\{\|u(x, 0)\|_{\mathcal{H}(0)}^{2}+\int_{0}^{t}\left(\|f\|^{2}+\langle g\rangle^{2}\right) d t+\int_{0}^{t}\|u(x, t)\|_{\mathcal{H}(t)}^{2} d t\right\}
\end{aligned}
$$

from which (2.17) follows by applying Lemma 2.1 by taking as

$$
\begin{aligned}
& \rho(t)=C^{\prime}\left\{\|u(x, 0)\|_{\mathcal{H}(0)}^{2}+\int_{0}^{t}\left(\|f\|^{2}+\langle g\rangle^{2}\right) d t\right\} \\
& \gamma(t)=\|u(x, t)\|_{\mathcal{H}(t)}^{2}+\int_{0}^{t} \ll u(x, t) \gg_{1}^{2} d t .
\end{aligned}
$$

It is necessary to hold (2.14) and (2.20) simultaneously and (2.16) satisfies the both conditions.

Q.E.D. 
Proposition 2.2. Suppose that (2.16) holds. For any solution $u(x, t) \in \mathcal{E}_{t}^{0}\left(H^{2}\left(R_{+}^{n}\right)\right) \cap \mathcal{E}_{t}^{1}\left(H^{1}\left(R_{+}^{n}\right)\right) \cap \mathcal{E}_{t}^{2}\left(L^{2}\left(R_{+}^{n}\right)\right)$ of $P(L, B)$, if

$$
\begin{aligned}
& L[u(x, t)]=f(x, t) \in H^{1}\left(R_{+}^{n} \times(0, T)\right) \\
& B[u(x, t)]=g(s, t) \in H^{1}\left(R^{n-1} \times(0, T)\right),
\end{aligned}
$$

then the trace of $u(x, t)$ to $R^{n-1} \times(0, T)$ is in $H^{2}\left(R^{n-1} \times(0, T)\right)$ and the following energy inequality holds

$$
\begin{aligned}
& \|u(x, t)\|_{2}^{2}+\int_{0}^{t} \ll u(x, t) \gg_{2}^{2} d t \\
\leqslant & C\left\{\|u(x, 0)\|_{2}^{2}+\left\|\frac{\partial u}{\partial t}(x, 0)\right\|_{1}^{2}+\|f(x, 0)\|^{2}\right. \\
& \left.+\int_{0}^{t}\left(\left\|\frac{\partial f}{\partial t}(x, t)\right\|^{2}+\sum_{j=1}^{n-1}\left\|\frac{\partial f}{\partial x_{j}}(x, t)\right\|^{2}+\ll g(s, t) \gg_{1}^{2}\right) d t\right\} .
\end{aligned}
$$

Proof. At first let us assume that not only $u(x, t)$ but also $\frac{\partial u}{\partial t}(x, t)$ and $\frac{\partial u}{\partial x_{j}}(x, t) \quad(j=1,2, \cdots, n-1)$ belong to $\mathcal{E}_{t}^{0}\left(H^{2}\left(R_{+}^{n}\right)\right)$ $\cap \mathcal{E}_{t}^{1}\left(H^{1}\left(R_{+}^{n}\right)\right) \cap \mathcal{E}_{t}^{2}\left(L^{2}\left(R_{+}^{n}\right)\right)$. The differentiation of (1.5) with respect to $t$ gives

$$
\begin{aligned}
& L\left[\frac{\partial u}{\partial t}(x, t)\right]=-L_{t}^{\prime}[u(x, t)]+f_{t}^{\prime}(x, t) \\
& B\left[\frac{\partial u}{\partial t}(x, t)\right]=-B_{t}^{\prime}[u(x, t)]+g_{t}^{\prime}\left(x^{\prime}, t\right),
\end{aligned}
$$

from the additional assumption Proposition 2.1 can be applied to $\frac{\partial u}{\partial t}(x, t)$, then we have

$$
\begin{aligned}
& \left\|\frac{\partial u}{\partial t}(x, t)\right\|_{\mathcal{H}(t)}^{2}+\int_{0}^{t}\left\langle\frac{\partial u}{\partial t}(x, t)\right\rangle_{1}^{2} d t \\
\leqslant & C\left\{\left\|\frac{\partial u}{\partial t}(x, 0)\right\|_{\mathcal{H}(0)}^{2}+\int_{0}^{t}\left(\left\|-L_{t}^{\prime}[u(x, t)]+f_{t}^{\prime}(x, t)\right\|^{2}\right.\right. \\
& \left.\left.+\left\langle-B_{t}^{\prime}[u(x, t)]+g_{t}^{\prime}(x, t)\right\rangle^{2}\right) d t\right\} .
\end{aligned}
$$

By the same manner we get

$$
\begin{aligned}
& \left.\left\|\frac{\partial u}{\partial x_{j}}(x, t)\right\|_{\mathcal{H}(t)}^{2}+\int_{0}^{t} \ll \frac{\partial u}{\partial x_{j}}(x, t)\right\rangle_{1}^{2} d t \\
\leqslant & C\left\{\left\|\frac{\partial u}{\partial x_{j}}(x, 0)\right\|_{\mathcal{H}(0)}^{2}+\int_{0}^{t}\left(\left\|-L_{x_{j}}^{\prime}[u(x, t)]+\frac{\partial f}{\partial x_{j}}(x, t)\right\|^{2}\right.\right. \\
& \left.\left.+\left\langle-B_{x_{j}}^{\prime}[u(x, t)]+\frac{\partial g}{\partial x_{j}}\left(x^{\prime}, t\right)\right\rangle^{2}\right) d t\right\}
\end{aligned}
$$


for $j=1,2, \cdots, n-1$. Remark that $\left\|L_{t}^{\prime}[u(x, t)]\right\|^{2}, \quad\left\|L_{x_{j}}^{\prime}[u(x, t)]\right\|^{2}$, $\left\langle B_{t}^{\prime}[u(x, t)]\right\rangle^{2}$ and $\left\langle B_{x j}^{\prime}[u(x, t)]\right\rangle^{2}$ are estimated by const. $\|u(x, t)\|_{2}^{2}$ and that from $L u=f$ we have

$$
\begin{aligned}
& \left\|\frac{\partial^{2} u}{\partial x_{n}^{2}}(x, t)\right\|^{2} \leqslant \text { const }\left(\sum_{i+j \neq 2 n}\left\|\frac{\partial^{2} u}{\partial x_{i} \partial x_{j}}(x, t)\right\|^{2}\right. \\
& \left.\quad+\left\|\frac{\partial u}{\partial t}(x, t)\right\|_{1}^{2}+\|f(x, t)\|^{2}\right) .
\end{aligned}
$$

On the other hand these inequalities are evident:

$$
\begin{aligned}
\|\| u(x, t)\|\|_{2}^{2} & \leqslant \operatorname{const}\left(\left\|\frac{\partial u}{\partial t}(x, t)\right\|_{\mathscr{H}(t)}^{2}+\sum_{j=1}^{n-1}\left\|\frac{\partial u}{\partial x_{j}}(x, t)\right\|_{\mathscr{H}(t)}^{2}\right. \\
+ & \left.\|u(x, t)\|_{\mathscr{H}(t)}^{2}+\left\|\frac{\partial^{2} u}{\partial x_{n}^{2}}(x, t)\right\|^{2}\right), \\
\ll u(x, t) \gg_{2}^{2} & \leqslant \operatorname{const}\left(\left\langle\frac{\partial u}{\partial t}(x, t)\right\rangle_{1}^{2}+\sum_{j=1}^{n-1}\left\langle\frac{\partial u}{\partial x_{j}}(x, t)\right\rangle_{1}^{2}\right. \\
+ & \left.\ll u(x, t) \gg_{1}^{2}\right) .
\end{aligned}
$$

Therefore by substituting into (2.19) the estimates (2.22) (2.23) (2.24) and using the above remark we get for some constant $C^{\prime}$

$$
\begin{aligned}
& \|u(x, t)\| \|_{2}^{2}+\int_{0}^{t} \ll u(x, t) \gg_{2}^{2} d t \\
\leqslant & C^{\prime}\left\{\|u(x, 0)\|_{2}^{2}+\|f(x, t)\|^{2}+\int_{0}^{t}\left(\left\|f^{\prime}(x, t)\right\|^{2}\right.\right. \\
& \left.+\sum_{j=1}^{n-1}\left\|\frac{\partial f}{\partial x_{j}}(x, t)\right\|^{2}+\ll g\left(x^{\prime}, t\right) \gg_{1}^{2}\right) d t \\
& \left.+\int_{0}^{t}\|u(x, t)\| \|_{2}^{2} d t\right\} .
\end{aligned}
$$

It is evident that

$$
\begin{aligned}
& \|u(x, 0)\| \|_{2}^{2} \leqslant \text { const }\left(\|u(x, 0)\|_{2}^{2}+\left\|\frac{\partial u}{\partial t}(x, 0)\right\|_{1}^{2}+\|f(x, 0)\|^{2}\right) \\
& \|f(x, t)\|^{2} \leqslant 2 T\left(\|f(x, 0)\|^{2}+\int_{0}^{t}\left\|\frac{\partial f}{\partial t}(x, t)\right\|^{2} d t\right) .
\end{aligned}
$$

Then it follows that for some constant $C^{\prime \prime}>0$ 


$$
\begin{aligned}
& \|u(x, t)\|_{2}^{2}+\int_{0}^{t} \ll u(x, t) \gg_{2}^{2} d t \\
\leqslant & C^{\prime \prime}\left\{\|u(x, 0)\|_{2}^{2}+\left\|\frac{\partial u}{\partial t}(x, 0)\right\|_{1}+\|f(x, 0)\|^{2}\right. \\
& +\int_{0}^{t}\left(\left\|\frac{\partial f}{\partial t}(x, t)\right\|^{2}+\sum_{j=1}^{n-1}\left\|\frac{\partial f}{\partial x_{j}}(x, t)\right\|^{2}+\ll g\left(x^{\prime}, t\right) \gg_{1}^{2} d t\right. \\
& \left.+\int_{0}^{t}\|u(x, t)\|_{2}^{2} d t\right\},
\end{aligned}
$$

from this (2.21) is derived only by the application of Lemma 2.1 by taking

$$
\begin{aligned}
\rho(t)= & C^{\prime \prime}\left\{\|u(x, 0)\|_{2}^{2}+\left\|\frac{\partial u}{\partial t}(x, 0)\right\|_{1}^{2}+\|f(x, 0)\|^{2}\right. \\
& \left.+\int_{0}^{t}\left(\left\|\frac{\partial f}{\partial t}(x, t)\right\|^{2}+\sum_{j=1}^{n-1}\left\|\frac{\partial f}{\partial x_{j}}(x, t)\right\|^{2}+\ll g\left(x^{\prime}, t\right) \gg_{1}^{2}\right) d t\right\} \\
\gamma(t)= & \|u(x, t)\|_{2}^{2}+\int_{0}^{t} \ll u(x, t) \gg_{2}^{2} d t .
\end{aligned}
$$

To complete the proof it is necessary to remove the additional assumption on the regularity of $u(x, t)$. For this purpose let us use the following mollifier with respect to $\left(x^{\prime}, t\right)$ :

Let $\varphi(l)$ be a positive $C^{\infty}$-function with a support contained in $[-2,-1]$ such that

$$
\int_{-\infty}^{\infty} \varphi(l) d l=1
$$

Define a mollifier $\varphi_{\delta_{\left(x^{\prime}, t\right)}^{*}}$ for $v(x, t) \in \mathcal{E}_{t}^{0}\left(H^{1}\left(R_{+}^{n}\right)\right)$ by

$$
\begin{aligned}
v_{\delta}(x, t)= & \left(\varphi_{\left.\delta_{\left(x^{\prime}, t\right)}^{*} v\right)\left(x^{\prime}, x_{n}, t\right)}\right. \\
& =\int_{R^{n-1}} d y^{\prime} \int_{-\infty}^{\infty} d \tau \varphi_{\delta}(t-\tau) \prod_{j=1}^{n-1} \varphi_{\delta}\left(x_{j}-y_{j}\right) v\left(y^{\prime}, x_{n}, \tau\right)
\end{aligned}
$$

where $\varphi_{\delta}(l)=\frac{1}{\delta} \varphi\left(\frac{l}{\delta}\right)$. Then for any non-negative multi-index $\alpha^{\prime}=\left(\alpha_{1}, \cdots, \alpha_{n-1}\right)$ and non-negative integer $j$

$$
\left(\frac{\partial}{\partial t}\right)^{j}\left(\frac{\partial}{\partial x^{\prime}}\right)^{\infty \prime} v_{\delta}(x, t) \in \mathcal{E}_{t}^{0}\left(H^{1}\left(R_{+}^{n}\right)\right) \text {. }
$$

For $w\left(x^{\prime}, t\right) \in \mathcal{E}_{t}^{0}\left(L^{2}\left(R^{n-1}\right)\right)$ we define $\varphi_{\delta_{\left(x^{\prime}, t\right)}^{*}}^{*}$ by the same manner, and we have for any $j$ and $\alpha^{\prime}$ 


$$
\left(\frac{\partial}{\partial t}\right)^{j}\left(\frac{\partial}{\partial x^{\prime}}\right)^{\alpha^{\prime}} w_{\delta}\left(x^{\prime}, t\right) \in \mathcal{E}_{t}^{0}\left(L\left(R^{n-1}\right)\right)
$$

Applying $\varphi_{\delta_{\left(x^{\prime}, t\right)}^{*}}^{*}$ to (1.5) we get

$$
\begin{aligned}
& L\left[u_{\delta}(x, t)\right]=f_{\delta}(x, t)-\left(C_{\delta} u\right)(x, t) \\
& B\left[u_{\delta}(x, t)\right]=g_{\delta}\left(x^{\prime}, t\right)-\left(\Gamma_{\delta} u\right)\left(x^{\prime}, t\right)
\end{aligned}
$$

where

$$
\begin{aligned}
& \left(C_{\delta} u\right)(x, t)=\left[\varphi_{\delta_{\left(x^{\prime}, t\right)}^{*}}^{*}, a_{1}(x, t: D)\right] \frac{\partial u}{\partial t}+\left[\varphi_{\delta_{\left(x^{\prime}, t\right)}^{*},} a_{2}(x, t: D)\right] u \\
& \left(\Gamma_{\delta} u\right)\left(x^{\prime}, t\right)=\left[\varphi_{\delta_{\left(x^{\prime}, t\right)}^{*}},\left(\frac{\partial}{\partial v_{t}}+\sigma_{2}\right)\right] u+\left[\varphi_{\delta_{\left(x^{\prime}, t\right)}^{*}, \sigma_{1}}\right] \frac{\partial u}{\partial t}
\end{aligned}
$$

for $t \in\left[0, T-2 \delta_{0}\right]$. Since $\frac{\partial u_{\delta}}{\partial x_{j}}(x, t)(j=1,2, \cdots, n-1)$ and $\frac{\partial u_{\delta}}{\partial t}(x, t)$ are in $\mathcal{E}_{t}^{0}\left(H^{2}\left(R_{+}^{n}\right)\right) \cap \mathcal{E}_{t}^{1}\left(H^{1}\left(R_{+}^{n}\right)\right) \cap \mathcal{E}_{t}^{2}\left(L^{2}\left(R_{+}^{n}\right)\right)$, the result obtained under the additional condition is applicable, then

$$
\begin{gathered}
\left.\left\|u_{\delta}(x, t)\right\|\right|_{2} ^{2}+\int_{0}^{t} \ll u_{\delta}(x, t) \gg_{2}^{2} d t \\
\leqslant C\left\{\left\|u_{\delta}(x, 0)\right\|_{2}^{2}+\left\|\frac{\partial u_{\delta}}{\partial t}(x, 0)\right\|^{2}+2\left\|f_{\delta}(x, 0)\right\|^{2}\right. \\
+2 \int_{0}^{t}\left(\left\|\frac{\partial f_{\delta}}{\partial t}(x, t)\right\|^{2}+\sum_{j=1}^{n-1}\left\|\frac{\partial f_{\delta}}{\partial x_{j}}(x, t)\right\|^{2}+\ll g_{\delta}\left(x^{\prime}, t\right) \gg_{1}^{2}\right) d t \\
+2\left\|\left(C_{\delta} u\right)(x, 0)\right\|+2 \int_{0}^{t}\left(\left\|\frac{\partial\left(C_{\delta} u\right)}{\partial t}(x, t)\right\|^{2}\right. \\
\left.\left.+\sum_{j=1}^{n-1}\left\|\frac{\partial\left(C_{\delta} u\right)}{\partial x_{j}}(x, t)\right\|^{2}+\ll\left(\Gamma_{\delta} u\right)\left(x^{\prime}, t\right) \gg_{1}^{2}\right) d t\right\} .
\end{gathered}
$$

Evidently it holds that for $t \in\left[0, T-\delta_{0}\right]\left(\delta_{0}>0\right)$

$$
\begin{array}{ll}
\left\|\left(\frac{\partial}{\partial t}\right)^{j} u_{\delta}(x, t)\right\|_{2-j} \longrightarrow\left\|\left(\frac{\partial}{\partial t}\right)^{j} u(x, t)\right\|_{2-j} & (j=0,1,2) \\
\left\|\left(\frac{\partial}{\partial t}\right)^{j}\left(\frac{\partial}{\partial x^{\prime}}\right)^{a^{\prime}} f_{\delta}(x, t)\right\| \longrightarrow\left\|\left(\frac{\partial}{\partial t}\right)^{j}\left(\frac{\partial}{\partial x^{\prime}}\right)^{a^{\prime}} f(x, t)\right\| & \left(j+\left|\alpha^{\prime}\right| \leqslant 1\right) \\
\ll g_{\delta}\left(x^{\prime}, t\right) \gg_{1} \longrightarrow \ll g\left(x^{\prime}, t\right) \gg_{1} &
\end{array}
$$

when $\delta$ tends to zero. Moreover we have

$$
\left\|\left(C_{\delta} u\right)(x, 0)\right\| \rightarrow 0
$$


(2.28) $\int_{0}^{T-\delta_{0}}\left(\left\|\frac{\partial}{\partial t}\left(C_{\delta} u\right)(x, t)\right\|^{2}+\sum_{j=1}^{n-1}\left\|\frac{\partial}{\partial x_{j}}\left(C_{\delta} u\right)(x, t)\right\|^{2}+\ll\left(\Gamma_{\delta} u\right) \gg_{1}^{2}\right) d t$

when $\delta$ tends to 0 . These proofs are essentially same as the estimates of commutators in the proof of Proposition 2.6 of [6]. With the aid of (2.27) (2.28) the passage to the limit of (2.26) when $\delta \rightarrow 0$ gives the required inequality for $u(x, t)$. Thus the proof is completed.

Q.E.D.

Energy inequality in the case when $\partial \Omega=S$ is compact. Let us define the functions $c_{j}(s, t)(j=1,2)$ on $S \times[0, T]$ by

$$
\begin{aligned}
c_{1}(s, t)= & \sum_{i, j=1}^{n} a_{i j}(s, t) \nu_{i}(s) \nu_{j}(s)+\left(\sum_{j=1}^{n} h_{j}(s, t) \nu_{j}(s)\right)^{2} \\
& +\left\{\left(\sum_{i, j=1}^{n} a_{i j}(s, t) \nu_{i}(s) \nu_{j}(s)\right)^{2}+\left(\sum_{j=1}^{n} h_{j}(s, t) \nu_{j}(s)\right)^{2}\right\} \\
& \times \sum_{i=1}^{n}\left(h_{i}(s, t)^{2}+\left(\sum_{j=1}^{n} a_{i j}(s, t) \nu_{j}(s)\right)^{2}\right) / d^{2}
\end{aligned}
$$

$$
\begin{aligned}
c_{2}(s, t)= & 2\left\{\left(\sum_{i, j=1}^{n} a_{i j}(s, t)^{2}+\sum_{i=1}^{n} h_{i}(s, t)^{2}\right) / d\right. \\
& \left.+\left|\sum_{i=1}^{n} h_{i}(s, t) \nu_{i}(s)\right|+1\right\}
\end{aligned}
$$

Remark that $\sum_{i=1}^{n}\left|\sum_{j=1}^{n} a_{i j} \nu_{j}-v_{i}\right|^{2}, \sum_{i=1}^{n} h_{i} \nu_{i}$ and these functions are invariant under any orthogonal transformation of variables, and the condition (2.3) is conserved under any transformation of variables.

Theorem 1. Suppose that

$$
b(s, t)<\frac{d}{4} \min \left(1, \sqrt{\frac{\alpha(s, t)}{c_{2}(s, t)}}, \frac{\alpha(s, t)}{\sqrt{c_{1}(s, t)+4 \alpha(s, t)^{2}}}\right)
$$

holds on $S \times[0, T]$. For any solution $u(x, t) \in \mathcal{E}_{t}^{2}\left(H^{2}(\Omega)\right) \cap \mathcal{E}_{t}^{1}\left(H^{1}(\Omega)\right)$ $\cap \mathcal{E}_{t}^{2}\left(L^{2}(\Omega)\right)$ of $P(L, B)$, if

$$
\begin{aligned}
& L[u(x, t)]=f(x, t) \in H^{1}(\Omega \times(0, T)) \\
& B[u(x, t)]=g(s, t) \in H^{1}(S \times(0 T)),
\end{aligned}
$$

the trace of $u(x, t)$ to $S \times(0, T)$ is in $H^{2}(S \times(0, T))$ and the energy inequality 


$$
\begin{gathered}
\|\| u(x, t)\|\|_{2, \Omega}^{2}+\int_{0}^{t} \ll u(x, t) \gg_{2, S}^{2} d t \\
\leqslant C\left\{\|u(x, 0)\|_{2, L^{2}(\Omega)}^{2}+\left\|\frac{\partial u}{\partial t}(x, 0)\right\|_{2, L^{2}(\Omega)}^{2}+\|f(x, 0)\|_{L^{2}(\Omega)}^{2}\right. \\
\left.\quad+\int_{0}^{t}\left(\|\| f(x, t) \|_{1, \Omega}^{2}+\ll g(s, t) \gg_{1, S}^{2}\right) d t\right\}
\end{gathered}
$$

holds.

Proof. For any $s_{0} \in S$ there exists a neighborhood $U$ of $s_{0}$ in $R^{n}$ and a smooth function $\psi(x)$ such that

$$
\left.\operatorname{grad} \psi(x)\right|_{x=s_{0}}=\nu\left(s_{0}\right)
$$

and $\psi(x)=0$ is an equation of $U \cap S$. From the above remark we may assume $\nu\left(s_{0}\right)=(0,0, \cdots,-1)$. Let us define a transformation $\Psi$ from $U$ onto some domain in $R^{n}$ by

$$
\begin{aligned}
& y_{n}=\psi(x) \\
& y_{i}=x_{i}-s_{i} \quad(i=1,2, \cdots, n-1),
\end{aligned}
$$

evidently we have

$$
\begin{aligned}
& \Psi(\Omega \cap U) \subset R_{+}^{n} \\
& \Psi(S \cap U) \subset \partial R_{+}^{n} \\
& \Psi\left(s_{0}\right)=0 .
\end{aligned}
$$

When a solution $u(x, t)$ of $P(L, B)$ has its support contained in $(\Omega \cap U) \times[0, T]$, the function $\tilde{u}(y, t)$ defined by $\tilde{u}(y, t)=\tilde{u}(\Psi(x), t)$ $=u(x, t)$ satisfies

$$
\left\{\begin{array}{l}
\tilde{L}[\tilde{u}(y, t)]=\tilde{f}(y, t) \\
\tilde{B}[\tilde{u}(y, t)]=\tilde{g}\left(y^{\prime}, t\right)
\end{array}\right.
$$

where

$$
\begin{aligned}
& \widetilde{L}= \frac{\partial^{2}}{\partial t^{2}}+2\left(\sum_{j=1}^{n} h_{j} \frac{\partial \psi}{\partial x_{j}} \frac{\partial}{\partial y_{n}}+\sum_{j=1}^{n-1} h_{i} \frac{\partial}{\partial y_{i}}\right) \frac{\partial}{\partial t} \\
& \quad-\left(\sum_{i, j=1}^{n} a_{i j} \frac{\partial \psi}{\partial x_{i}} \frac{\partial \psi}{\partial x_{j}} \frac{\partial^{2}}{\partial y_{n}^{2}}+\sum_{i, j=1}^{n} a_{i j} \frac{\partial \psi}{\partial x_{j}} \frac{\partial^{2}}{\partial y_{i} \partial y_{n}}+\sum_{i, j=1}^{n-1} a_{i j} \frac{\partial^{2}}{\partial y_{i} \partial y_{j}}\right) \\
& \quad+\text { first order } \\
& \widetilde{B}=\sum_{i=1}^{n-1} v_{i} \frac{\partial}{\partial y_{i}}+\sum_{i=1}^{n} v_{i} \frac{\partial \psi}{\partial x_{i}} \frac{\partial}{\partial y_{n}}-\sigma_{1} \frac{\partial}{\partial t}+\sigma_{2} .
\end{aligned}
$$


Since $(\operatorname{grad} \psi)\left(s_{0}\right)=(0,0, \cdots,-1)$ and $\nu\left(y^{\prime}, 0\right)=(0,0, \cdots,-1)$, by taking account of the continuity of $\operatorname{grad} \psi, b(s, t)$ and $\alpha(s, t)$ we have for some neighborhood $U_{0}$ of $s_{0}$ and $T^{\prime}>0$ that

$$
\begin{aligned}
& \sup _{\left(y^{\prime}, t\right) \in \mathbb{\Psi}\left(U_{0} \cap S\right) \times\left[0, T^{\prime},\right.} \tilde{b}\left(y^{\prime}, t\right) \\
& <\min \left(1, \sqrt{\inf _{\frac{\left(y^{\prime}, t\right)}{\operatorname{iup}_{\left(y^{\prime}, t\right)} \hat{\alpha}\left(y^{\prime}, t\right)}} \widetilde{c}_{2}\left(y^{\prime}, t\right)}, \frac{\inf \hat{\alpha}\left(y^{\prime}, t\right)}{\sqrt{\sup \widetilde{c}_{1}\left(y^{\prime}, t\right)+4 \sup \hat{\alpha}\left(y^{\prime}, t\right)^{2}}}\right)
\end{aligned}
$$

where $\tilde{b}\left(y^{\prime}, t\right)$ and $\widetilde{c}_{i}(y, t)$ denote the quantities for $\widetilde{L}$ and $\widetilde{B}$ defined by (2.5), (2.29) and (2.30). Therefore when only the solutions with the support contained in $\left(U_{0} \cap \Omega\right) \times\left[0, T^{\prime}\right]$ are considered, we can say that $\widetilde{L}$ and $\widetilde{B}$ satisfy the condition (2.16) and Proposition 2.2 can be applied for (2.24), then the energy inequality (2.21) holds for $\tilde{u}(y, t)$, therefore (2.33) also holds for $u(x, t)$.

Let $\left\{\Phi_{j}\right\}_{j=1}^{m}$ be a partition of unity of a neighborhood of $S$ such that $\sum_{j=1}^{m} \Phi_{j}^{2}=1$ in a neighborhood of $S$ and the support of $\Phi_{j}(x)$ is contained in a neighborhood of a point $s_{j} \in S$ with the above properties.

Put $u_{j}(x, t)=\Phi_{j}(x) u(x, t)$, then

$$
\begin{aligned}
& L\left[u_{j}(x, t)\right]=\Phi_{j}(x) f(x, t)-\left[L, \Phi_{j}\right] u(x, t) \\
& B\left[u_{j}(x, t)\right]=\Phi_{j}(s) g(s, t)-\left[B, \Phi_{j}\right] u(s, t)
\end{aligned}
$$

and we get for $t \in\left[0, T_{j}^{\prime}\right]$

$$
\begin{aligned}
& \left.\quad\left\|u_{j}(x, t)\right\|\right|_{2, \Omega} ^{2}+\int_{0}^{t} \ll u_{j}(x, t) \gg_{2, \mathrm{~S}}^{2} d t \\
& \leqslant C_{j}\left\{\left\|u_{j}(x, 0)\right\|_{2, L^{2}(\Omega)}^{2}+\left\|\frac{\partial u_{j}}{\partial t}(x, 0)\right\|_{1, L^{2}(\Omega)}^{2}\right. \\
& \quad+\left\|\Phi_{j} f(x, 0)-\left[L, \Phi_{j}\right] u(x, 0)\right\|^{2} \\
& \left.\quad+\int_{0}^{t}\left(\|\| \Phi_{j} f(x, t)-\left[L, \Phi_{j}\right] u \|_{1, \Omega}^{2}+\ll \Phi_{j} g-\left[B, \Phi_{j}\right] u \gg_{1, S}^{2}\right) d t\right\}
\end{aligned}
$$

here $C_{j}$ is determined by $L, B$ and $\Phi_{j}$. And for $\left(1-\sum_{j=1}^{m} \Phi_{j}^{2}\right)^{1 / 2} u(x, t)$ (2.33) holds since its support does not joint with $S$. By summing up (2.35) and using

$$
\begin{aligned}
& \|\left[L\left[L, \Phi_{j}\right] u\|\|_{1} \leqslant \text { const }\|u\| \|_{2}\right. \\
& \ll\left[B, \Phi_{j}\right] u \gg_{1} \leqslant \text { const }\|u\| \|_{2},
\end{aligned}
$$


we have

$$
\begin{aligned}
& \quad\|u(x, t)\| \|_{2, \Omega}^{2}+\int_{0}^{t} \ll u(x, t) \gg_{2, \mathrm{~S}}^{2} d t \\
& \leqslant C\left\{\|u(x, 0)\|_{2, L^{2}(\Omega)}^{2}+\left\|\frac{\partial u}{\partial t}(x, 0)\right\|_{1, L^{2}(\Omega)}^{2}+\|f(x, 0)\|_{L^{2}(\Omega)}^{2}\right. \\
& \left.\quad+\int_{0}^{t}\left(\|\| f\|\|_{1}^{2}+\ll g \gg_{1}^{2}\right) d t+\int_{0}^{t}\|\| u\|\|_{2}^{2} d t\right\}
\end{aligned}
$$

and by applying Lemma 2.1 we get (2.33) for $t \in\left[0, T_{0}^{\prime}\right]$ where $T_{0}^{\prime}=\min _{j} T_{j}^{\prime}$. From the uniform continuity of the functions which determin $T_{0}^{\prime}$, there is no difficulty to show that (2.33) holds for all $t \in[0, T]$.

\section{$\S 3$. Existence of the Solution}

Hereafter assume that $\Omega$ is a domain with a compact boundary. We state the theorem of existence of the solution.

Theorem 2. Suppose that the condition (2.32) holds. For any initial data $\left\{u_{0}(x), u_{1}(x)\right\} \in H^{2}(\Omega) \times H^{1}(\Omega)$, second member $f(x, t) \in$ $H^{1}(\Omega(0, T))$ and boundary data $g(s, t) \in H^{1}(S \times(0, T))$, if the compatibility condition at $t=0$ of order 0

$$
\frac{\partial}{\partial v_{0}} u_{0}(x)-\sigma_{1}(s, 0) u_{1}(x)+\sigma_{2}(s, 0) u_{0}(x)=g(s, 0)
$$

is satisfied, then there exists a solution $u(x, t)$ of $P(L, B)$ uniquely in $\mathcal{E}_{t}^{0}\left(H^{2}(\Omega)\right) \cap \mathcal{E}_{t}^{1}\left(H^{1}(\Omega)\right) \cap \mathcal{E}_{t}^{2}\left(L^{2}(\Omega)\right)$.

At first let us prove

Lemma 3.1. Let

$$
\begin{aligned}
W & =\frac{\partial}{\partial w_{t}}-\sigma_{1} \frac{\partial}{\partial t}+\sigma_{2} \\
Z & =\frac{\partial}{\partial z_{t}}-\sigma_{1} \frac{\partial}{\partial t}+\sigma_{2}
\end{aligned}
$$

be two boundary operators which satisfy (2.3). Assume that for $P(L, W)$ the energy inequality (2.33) holds with a constant $C$ and that the existence of the solution is already known. If

$$
\sup _{s \times[0, T]}\left(\sum_{i=1}^{n}\left|v_{i}(s, t)-w_{i}(s, t)\right|^{2}\right)<\frac{1-\varepsilon_{0}}{C}
$$


holds for $\varepsilon_{0}>0$, then the existence of solution of $P(L, Z)$ follows.

Proof. Assume that $\left\{u_{0}(x), u_{1}(x)\right\} \in H^{3}(\Omega) \times H^{2}(\Omega)$ and the given data satisfy the compatibility condition for $Z$, namely

$$
\frac{\partial}{\partial z_{0}} u_{0}(x)-\sigma_{1}(s, 0) u_{1}(x)+\sigma_{2}(s, 0) u_{0}(x)=g(s, 0) \text {. }
$$

Let us construct $u^{(j)}(x, t) \in \mathcal{E}_{t}^{0}\left(H^{2}(\Omega)\right) \cap \mathcal{E}_{t}^{1}\left(H^{1}(\Omega)\right) \cap \mathcal{E}_{t}^{2}\left(L^{2}(\Omega)\right)(j=1,2, \cdots)$ successively by the following way: $u^{(1)}(x, t)$ be the solution

$$
\left\{\begin{aligned}
L\left[u^{(1)}(x, t)\right] & =f(x, t) \\
W\left[u^{(1)}(x, t)\right] & =g(s, t)-\left(\frac{\partial}{\partial z_{t}}-\frac{\partial}{\partial w_{t}}\right) u_{0}(x) \\
u^{(1)}(x, 0) & =u_{0}(x) \\
\frac{\partial u^{(1)}}{\partial t}(x, 0) & =u_{1}(x),
\end{aligned}\right.
$$

its existence is assured by the assumption that the existence of the solution of $P(L, W)$ because the compatibility condition for $W$ is satisfied from (3.3), and $g(s, t)-\left(\frac{\partial}{\partial z_{t}}-\frac{\partial}{\partial w_{t}}\right) u_{0}(x) \in H^{1}(S \times(0, T))$. We can define $u^{(j)}(x, t)$ for $j \geqslant 2$ succesively by the formula

$$
\left\{\begin{aligned}
L\left[u^{(j)}(x, t)\right] & =f(x, t) \\
B\left[u^{(j)}(x, t)\right] & =g(s, t)-\left(\frac{\partial}{\partial z_{t}}-\frac{\partial}{\partial w_{t}}\right) u^{(j-1)}(x, t) \\
u^{(j)}(x, 0) & =u_{0}(x) \\
\frac{\partial u^{(j)}}{\partial t}(x, 0) & =u_{1}(x)
\end{aligned}\right.
$$

since from $\left.u^{(j-1)}(x, t)\right|_{s \times[0, T]} \in H^{2}(S \times(0, T))$ and the compatibility condition for $W$ is satisfied.

The sequence $u^{(j)}(x, t) \quad(j=1,2, \cdots)$ converges in $\mathcal{E}_{t}^{0}\left(H^{2}(\Omega)\right)$ $\cap \mathcal{E}_{t}^{1}\left(H^{1}(\Omega)\right) \cap \mathcal{E}_{t}^{2}\left(L^{2}(\Omega)\right)$, indeed

$$
\begin{aligned}
L\left[u^{(j+1)}-u^{(j)}\right] & =0 \\
W\left[u^{(j+1)}-u^{(j)}\right] & =-\left(\frac{\partial}{\partial z_{t}}-\frac{\partial}{\partial w_{t}}\right)\left(u^{(j)}-u^{(j-1)}\right) \\
\left(u^{(j+1)}-u^{(j)}\right)(x, 0) & =0 \\
\frac{\partial}{\partial t}\left(u^{(j+1)}-u^{(j)}\right)(x, 0) & =0
\end{aligned}
$$


for $j \geqslant 2$ and the application of the energy inequality gives

$$
\begin{aligned}
& \left\|u^{(j+1)}(x, t)-u^{(j)}(x, t)\right\|_{2, Q}^{2}+\int_{0}^{t} \ll u^{(j+1)}(x, t)-u^{(j)}(x, t) \gg_{2, S}^{2} d t \\
\leqslant & \left.C \int_{0}^{t} \ll\left(\frac{\partial}{\partial z_{t}}-\frac{\partial}{\partial w_{t}}\right)\left(u^{(j)}(x, t)-u^{(j-1)}(x, t)\right)\right\rangle_{1, S}^{2} d t \\
\leqslant & C \int_{0}^{t} \sum_{i=1}^{n}\left|w_{i}-z_{i}\right|^{2} \ll u^{(j)}(x, t)-u^{(j-1)}(x, t) \gg_{2, S}^{2} d t
\end{aligned}
$$

from (3.2)

$$
\leqslant\left(1-\varepsilon_{0}\right) \int_{0}^{t} \ll u^{(j)}(x, t)-u^{(j-1)}(x, t) \gg_{2, \mathrm{~S}}^{2} d t .
$$

Thus we get for all $j \geqslant 2$

$$
\begin{aligned}
& \|\| u^{(j+1)}(x, t)-u^{(j)}(x, t) \|_{2, \Omega}^{2}+\int_{0}^{t} \ll u^{(j+1)}(x, t)-u^{(j)}(x, t) \gg_{2, \mathrm{~S}}^{2} d t \\
& \quad \leqslant \operatorname{const}\left(1-\varepsilon_{0}\right)^{j},
\end{aligned}
$$

which assures the convergence of $u^{(j)}(x, t)$ in $\mathcal{E}_{t}^{0}\left(H^{2}(\Omega)\right) \cap \mathcal{E}_{t}^{1}\left(H^{1}(\Omega)\right)$ $\cap \mathcal{E}_{t}^{2}\left(L^{2}(\Omega)\right)$. Denote its limit by $u(x, t)$ then we see from the passage to the limit of (3.4) that $u(x, t)$ satisfies

$$
\left\{\begin{aligned}
L[u(x, t)] & =f(x, t) \\
W[u(x, t)] & =g(s, t)-\left(\frac{\partial}{\partial z_{t}}-\frac{\partial}{\partial w_{t}}\right) u(s, t) \\
u(x, 0) & =u_{0}(x) \\
\frac{\partial u}{\partial t}(x, 0) & =u_{1}(x) .
\end{aligned}\right.
$$

This shows that $u(x, t)$ is the required solution.

For $\left\{u_{0}(x), u_{1}(x)\right\} \in H^{2}(\Omega) \times H^{1}(\Omega)$ if we get sequences $\left\{u_{0 k}(x)\right.$, $\left.u_{1 k}(x)\right\} \in H^{3}(\Omega) \times H^{2}(\Omega)$ and $g_{k}(s, t) \in H^{2}(S \times(0, T))(k=1,2, \cdots)$ such that

$$
\frac{\partial u_{0 k}}{\partial z_{0}}(x)-\sigma_{1}(s, 0) u_{1 k}(x)+\sigma_{2}(s, 0) u_{0 k}(x)=g_{k}(s, 0)
$$

and

$$
\begin{array}{lll}
u_{0 k} \longrightarrow u_{0} & \text { in } & H^{2}(\Omega) \\
u_{1 k} \longrightarrow u_{1} & \text { in } & H^{1}(\Omega) \\
g_{k} \longrightarrow g & \text { in } & H^{1}(S \times(0, T))
\end{array}
$$

when $k$ increases infinitely, there exists the solution $u^{(k)}(x, t)$ of 


$$
\left\{\begin{aligned}
L\left[u^{(k)}(x, t)\right] & =f(x, t) \\
Z\left[u^{(k)}(x, t)\right] & =g_{k}(s, t) \\
u^{(k)}(x, 0) & =u_{0 k}(x) \\
\frac{\partial u^{(k)}}{\partial t}(x, 0) & =u_{1 k}(x)
\end{aligned}\right.
$$

from the just obtained result. And by applying the energy inequality for $u^{(k)}-u^{(m)}$, we have for any $k, m$

$$
\begin{aligned}
& \left\|u^{(k)}(x, t)-u^{(m)}(x, t)\right\|_{2, \Omega}^{2}+\int_{0}^{t} \ll u^{(k)}(x, t)-u^{(m)}(x, t) \gg_{2, S}^{2} d t \\
\leqslant & \left\{C\left\|u_{0 k}(x)-u_{0 m}(x)\right\|_{2, L^{2}(\Omega)}^{2}+\left\|u_{1 k}(x)-u_{1 m}(x)\right\|_{1, L^{2}(\Omega)}^{2}\right. \\
& \left.+\int_{0}^{t} \ll g_{k}(s, t)-g_{m}(s, t) \gg_{1, S}^{2} d t\right\} .
\end{aligned}
$$

We see the convergence of $u^{(k)}(x, t)$ in $\mathcal{E}_{t}^{0}\left(H^{2}(\Omega)\right) \cap \mathcal{E}_{t}^{1}\left(H^{1}(\Omega)\right)$ $\cap \mathcal{E}_{t}^{2}\left(L^{2}(\Omega)\right)$ and the passage to the limit of (3.5) when $k \rightarrow \infty$ proves that the limit of $u^{(k)}(x, t)$ is the required solution for $\left\{u_{0}, u_{1}\right\}$ and $g$.

Now let us see the existence of sequences with the above properties. It is evident that there exists sequences $\left\{p_{0 k}, p_{1 k}\right\} \in H^{3}(\Omega)$ $\times H^{2}(\Omega)$ and $g_{k}(s, t) \in H^{2}(S \times(0, T))$ such that

$$
\begin{aligned}
& p_{0 k}(x) \rightarrow u_{0}(x) \quad \text { in } \quad H^{2}(\Omega) \\
& p_{1 k}(x) \rightarrow u_{1}(x) \quad \text { in } H^{1}(\Omega) \\
& g_{k}(s, t) \rightarrow g(s, t) \quad \text { in } \quad H^{1}(S \times(0, T)) \text {. }
\end{aligned}
$$

Then

$$
q_{k}(s)=\frac{\partial}{\partial z_{0}} p_{0 k}(x)-\sigma_{1}(s, 0) p_{1 k}(x)+\sigma_{2}(s, 0) p_{0 k}(x)-g_{k}(s, 0)
$$

is in $H^{1+1 / 2}(S)$ and $\left\langle q_{k}(s)\right\rangle_{1 / 2}$ tends to zero when $k$ increases infinitely since $\left\{u_{0}, u_{1}\right\}$ and $g$ satisfies the compatibility condition (3.3). Let $\Omega$ be the interior domain and $r_{k}(x)$ be a solution of a boundary value problem

$$
\begin{cases}\left(-\Delta+\lambda_{0}\right) r_{k}(x)=0 & \text { in } \Omega \\ \left(\frac{\partial}{\partial z_{0}}+\sigma_{2}(s, 0)\right) r_{k}(x)=q_{k}(s) & \text { on } S .\end{cases}
$$

When $\lambda_{0}$ is sufficiently large (3.6) has a unique solution in $H^{3}(\Omega)$ and the following estimate holds 


$$
\left\|r_{k}(x)\right\|_{2, L^{2}(\Omega)}^{2} \leqslant \text { const }\left\langle q_{k}(s)\right\rangle_{1 / 2}^{2} .
$$

Then if we define $\left\{u_{0 k}, u_{1 k}\right\}$ by

$$
\begin{aligned}
& u_{0 k}=p_{0 k}+r_{k} \\
& u_{1 k}=p_{1 k}
\end{aligned}
$$

$\left\{u_{0 k}, u_{1 k}\right\}$ and $g_{k}$ are the required ones. When $\Omega$ is an exterior domain, take a sphere $S_{1}$ containing $S$ in its interior and denote by $\Omega_{1}$ the domain surrounded by $S$ and $S_{1}$. We define $r_{k}(x)$ as the solution of

$$
\begin{array}{rlrl}
\left(-\Delta+\lambda_{0}\right) r_{k}(x) & =0 & \text { in } \quad \Omega_{1} \\
\left(\frac{\partial}{\partial z_{0}}+\sigma_{2}(s, 0)\right) r_{k}(x) & =q_{k}(s) & \text { on } S \\
\left(\frac{\partial}{\partial z_{0}}+\sigma_{2}(s, 0)\right) r_{k}(x) & =0 & & \text { on } S_{1} .
\end{array}
$$

Since $\Omega_{1}$ is compact, we have a unique solution $r_{k}(x) \in H^{3}(\Omega)$ and the estimate

$$
\left\|r_{k}(x)\right\|_{2, L^{2}(\boldsymbol{\Omega})}^{2} \leqslant \text { const }\left\langle q_{k}\right\rangle_{1 / 2}^{2} .
$$

Let $\mu(x)$ be a $C^{\infty}$-function such that $\operatorname{supp}(\mu(x)) \subset$ interior of $S_{1}$ and $\mu(x)$ equals 1 in a neighborhood of $S$. Then $\mu(x) r_{k}(x)$ is in $H^{3}(\Omega)$ and $\left\|\mu(x) r_{k}(x)\right\|_{2}$ tends to zero when $k$ increases infinitely. Therefore if we take $u_{0 k}, u_{1 k}$ as

$$
\begin{aligned}
& u_{0 k}(x)=\mu(x) r_{k}(x)+p_{0 k}(x) \\
& u_{1 k}(x)=p_{1 k}(x),
\end{aligned}
$$

these are the required ones. Thus the proof of lemma is completed.

Now we prove Theorem. At first remark that when $v(s, t)$ $=n(s, t)$ the existence of the solution is already proved (Theorem 1 of [8]). Let $B_{\eta}(\eta \in[0,1])$ be the boundary operator defined by

$$
B_{\eta}=\frac{\partial}{\partial n_{t}}-\eta\left(\frac{\partial}{\partial n_{t}}-\frac{\partial}{\partial v_{t}}\right)-\sigma_{1}(s, t) \frac{\partial}{\partial t}+\sigma_{2}(s, t) .
$$

Since for any $\eta \in[0,1] P\left(L, B_{\eta}\right)$ satisfies the condition (2.32), then the energy inequality (2.33) for $P\left(L, B_{\eta}\right)$ holds for any $\eta \in[0,1]$, moreover the constant $C$ can be taken independently of $\eta$. Since the existence of the solution for $P\left(L, B_{0}\right)$ is assured, by applying 
Lemma 3.1 by taking $W=B_{0}$ and $Z=B_{\eta}$ for $\eta \in\left[0, \varepsilon_{0}\right]\left(0<\varepsilon_{0}<\frac{1}{C}\right)$ we see the existence of the solution for $P\left(L, B_{\eta}\right)$ for $\eta \in\left[0, \varepsilon_{0}\right]$. Now apply Lemma 3.1 once more by taking $W=B_{\varepsilon_{0}}$ and $Z=B_{\eta}$ for $\eta \in\left[\varepsilon_{0}, 2 \varepsilon_{0}\right]$, the existence of the solution for $P\left(L, B_{\eta}\right)$ for $\eta \in\left[\varepsilon_{0}, 2 \varepsilon_{0}\right]$ is proved. Step by step for any $\eta \in[0,1]$ the existence of the solution for $P\left(L, B_{\eta}\right)$ is assured. Thus we get the existence of the solution of $P(L, B)$.

\section{$\S 4$. Regularity of the Solution}

The solution of $P(L, B)$ becomes regular according to the regularities of the initial data, second member and the boundary data. Since this equation is hyperbolic the given data must satisfy the compatibility condition of higher order. Suppose $u(x, t)$ $\in \mathcal{E}_{t}^{0}\left(H^{3}(\Omega)\right) \cap \mathcal{E}_{t}^{1}\left(H^{2}(\Omega)\right) \cap \mathcal{E}_{t}^{2}\left(H^{1}(\Omega)\right) \cap \mathcal{E}_{t}^{3}\left(L^{2}(\Omega)\right)$, then

$$
\begin{aligned}
\frac{\partial^{2} u}{\partial t^{2}}(x, 0) & =-a_{1}(x, 0: D) \frac{\partial u}{\partial t}(x, 0)-a_{2}(x, 0: D) u(x, 0)+f(x, 0) \\
& =-a_{1}(x, 0: D) u_{1}(x)-a_{2}(x, 0: D) u_{0}(x)+f(x, 0) .
\end{aligned}
$$

On the other hand the differentiation of $B u=g$ with respect to $t$ gives

$$
B\left[\frac{\partial u}{\partial t}(x, t)\right]+B[u(x, t)]=g^{\prime}(s, t)
$$

and if we put $t=0$ and substitute the above relation $u_{0}, u_{1}, g$ and $f$ should satisfy

$$
\begin{aligned}
& \frac{\partial}{\partial v_{0}} u_{1}(x)-\sigma_{1}(s, 0)\left(-a_{1} u_{1}-a_{2} u_{0}+f(x, 0)\right) \\
& \quad+\sigma_{2}(s, 0) u_{1}+\left(\frac{\partial}{\partial v_{t}}\right)_{t=0}^{\prime} u_{0}-\left(\sigma_{1}\right)_{t=0}^{\prime} u_{1}+\left(\sigma_{2}\right)_{t=0}^{\prime} u_{0}=g^{\prime}(s, 0) .
\end{aligned}
$$

We say that $u_{0}, u_{1}, f, g$ satisfy the compatibility condition of order 1 if (3.1) and (4.1) hold. The compatibility condition of higher order can be defined by the same manner:

Definition 4.1. The data $\left\{u_{0}, u_{1}\right\} \in H^{m+2}(\Omega) \times H^{m+1}(\Omega), g(s, t)$ $\in H^{m+1}(S \times(0, T))$ and $f(x, t) \in H^{m+1}(\Omega \times(0, T))$ are said to satisfy the compatibility condition of order $m$ when 


$$
\begin{gathered}
\sum_{k=0}^{p}\left(\begin{array}{c}
p \\
k
\end{array}\right)\left\{\left(\frac{\partial}{\partial v_{t}}\right)^{(k)} u_{p-k}-\left(\sigma_{1}(s, t)\right)_{t}^{(k)} u_{p-k+1}\right. \\
\left.+\left(\sigma_{2}(s, t)\right)_{t}^{(k)} u_{p-k}\right\}_{t=0}=g^{(p)}(x, 0)
\end{gathered}
$$

holds for $p=0,1,2, \cdots, m$ where $u_{p}(x)(p \geqslant 2)$ is defined successively by the formula

$$
\begin{aligned}
u_{p}(x)= & -\sum_{k=0}^{p-2}\left(\begin{array}{c}
p-2 \\
k
\end{array}\right)\left\{a(x, 0: D) u_{p-k-1}+a(x, 0: D) u_{p-k-2}\right\} \\
& +f^{(p-2)}(x, 0)
\end{aligned}
$$

Theorem 3. When the given data satisfy the compatibility condition of order $m$ the solution $u(x, t)$ of $P(L, B)$ belongs to $\mathcal{E}_{t}^{0}\left(H^{m+2}(\Omega)\right) \cap \mathcal{E}_{t}^{1}\left(H^{m+1}(\Omega)\right) \cap \cdots \cap \mathcal{E}_{t}^{m+2}\left(L^{2}(\Omega)\right)$.

If we use the following lemma, the proof is completely same as that of Theorem 2 of the previous paper [8].

Lemma 4.1. Suppose that $u(x, t) \in \mathcal{E}_{t}^{k}\left(H^{2}(\Omega)\right)$ satisfies

$$
\begin{aligned}
& a_{2}(x, t: D) u(x, t) \in \mathcal{E}_{t}^{k}\left(H^{p}(\Omega)\right) \\
& \left(\frac{\partial}{\partial v_{t}}-\sigma_{2}(s, t)\right) u(x, t) \in \mathcal{E}_{t}^{k}\left(H^{p+1 / 2}(S)\right.
\end{aligned}
$$

where $p$ and $k$ are non-negative integers, then

$$
u(x, t) \in \mathcal{E}_{t}^{k}\left(H^{p+2}(\Omega)\right) .
$$

One can also prove this lemma by the same way as that of Lemma 3.5 of [6] (page 604). The difference is only that the boundary operator of the previous paper is $\partial / \partial n_{t}+\sigma_{2}$ and that of this case is $\partial / \partial v_{t}+\sigma_{2}$. But since the a priori estimate of an elliptic operator

$$
\begin{aligned}
\|w\|_{p+2}^{2} \leqslant & K_{p}\left\{\left\|a_{2}(x, t: D) w\right\|_{p}^{2}+\|w\|^{2}\right. \\
& \left.+\left\langle\frac{\partial}{\partial v_{t}}+\sigma_{2}(s, t) w^{2}\right\rangle_{p+1 / 2}^{2}\right\}
\end{aligned}
$$

holds, we get Lemma 4.1 by the same proof.

\section{$\S 5$. Dependence Domain}

One of the important properties of Cauchy problem for 
hyperbolic equations is the existence of a dependence domain. This means that a hyperbolic equation is an equation of propagation phenomenon with a finite velocity. Concerning the mixed problems for hyperbolic equations the existence of a dependence domain is not a trivial fact deduced from the results of Cauchy problem. As indicated by $R$. Hersh [4] if we set some boundary condition a mixed problem for the wave equation has an infinite velocity.

Our actual problem $P(L, B)$ has a finite velocity.

Let $\lambda_{1}(x, t: \xi), \lambda_{2}(x, t: \xi)$ be the roots of the characteristc equation of $L$

$$
\lambda^{2}+2 \sum_{i=1}^{n} h_{i}(x, t) \xi_{i} \lambda-\sum_{i, j=1}^{n} a_{i j}(x, t) \xi_{i} \xi_{j}=0
$$

for $(x, t) \in \bar{\Omega} \times[0, T]$ and $\xi \in R^{n}$. Denote

$$
\lambda_{\max }=\sup _{\substack{|\xi|=1, i=1,2 \\(x, t) \in \bar{\Omega} \times[0, \pi]}}\left|\lambda_{i}(x, t: \xi)\right| .
$$

For $x_{0} \in \bar{\Omega}$ we denote by $C\left(x_{0}, t_{0}\right)$ the backward cone with a vertex $\left(x_{0}, t_{0}\right)$ defined by

$$
\left\{(x, t) ;\left|x-x_{0}\right|=\lambda_{\max }\left(t_{0}-t\right)\right\},
$$

and by $\Lambda\left(x_{0}, t_{0}\right)$ the interior of $C\left(x_{0}, t_{0}\right)$.

Theorem 4. There exists a smooth positive function $\gamma(s, t)$ on $S \times[0, T]$ determined by $L$. Suppose

$$
b(s, t)<\gamma(s, t) \frac{d}{4} \min \left(1, \sqrt{\frac{\alpha(s, t)}{c_{2}(s, t)}}, \frac{\alpha(s, t)}{\sqrt{c_{1}(s, t)+4 \alpha(s, t)^{2}}}\right)
$$

holds on $S \times[0, T]$. Let $u(x, t)$ be a $C^{2}$-solution of $P(L, B)$ defined in $\Lambda\left(x_{0}, t_{0}\right) \cap(\Omega \times[0, T])$. If $u_{0}(x), u_{1}(x)$ are zero in $\Lambda\left(x_{0}, t_{0}\right)$ $\cap\{\bar{\Omega}, t=0\}, g(s, t)$ is zero in $\Lambda\left(x_{0}, t_{0}\right) \cap S \times[0, T]$, and $f(x, t)$ is zero in $\Lambda\left(x_{0}, t_{0}\right) \cap(\Omega \times[0, T])$, then $u(x, t)$ is identically zero in $\Lambda\left(x_{0}, t_{0}\right)$ $\cap(\Omega \times[0, T])$.

Proof. We use the method of sweeping out of F. John. Define for $0<\theta<\lambda_{\max }^{2} t_{0}^{2}$

$$
\varphi_{\theta}(x, t)=\left(t_{0}-t\right)-\frac{1}{\lambda_{\max }} \sqrt{\left|x-x_{0}\right|^{2}+\theta}
$$

and 


$$
C_{\theta}\left(x_{0}, t_{0}\right)=\left\{(x, t) ; \varphi_{\theta}(x, t)=0\right\} .
$$

If the local uniqueness of the solution, especially near the boundary, is shown under the condition (2.23), it suffices to show the following:

After the change of variables

$$
\begin{aligned}
t^{\prime} & =\varphi_{\theta}(x, t) \\
x^{\prime} & =x
\end{aligned}
$$

(1.5) transformed into

$$
\begin{aligned}
& L_{\theta}[\tilde{u}]=0 \\
& B_{\theta}[\tilde{u}]=0
\end{aligned}
$$

where $\tilde{u}\left(x^{\prime}, t^{\prime}\right)=u(x, t)$. Then the condition (2.32) corresponding to $L_{\theta}, B_{\theta}$ will be satisfied for all $\theta \in\left(0, \lambda_{\max } t_{0}^{2}\right)$ when (5.2) is fulfiled.

Let

$$
\Phi_{\theta}=\left(1+2 \sum_{i=1}^{n} h_{i}(x, t) \frac{\partial \varphi_{\theta}}{\partial x_{i}}-\sum_{i, j=1}^{n} a_{i j}(x, t) \frac{\partial \varphi_{\theta}}{\partial x_{i}} \frac{\partial \varphi_{\theta}}{\partial x_{j}}\right)
$$

and we have for all $\theta \in\left(0, \lambda_{\max } t_{0}^{2}\right)$ and $(s, t) \in S \times[0, T]$

$$
\Phi_{\theta}(s, t) \in(0, \delta) \quad(\delta<+\infty) .
$$

By applying (2.29) (2.30) and (2.5) we have $c_{i}(s, t: \theta)(i=1,2)$ and $b(s, t: \theta)$ for $L_{\theta}$ and $B_{\theta}$ are given by

$$
\begin{aligned}
& c_{1}(s, t: \theta)=\Phi_{\theta}^{-1} \sum_{i, j=1}^{n} a_{i j}(s, t) \nu_{i} \nu_{j}+\Phi_{\theta}^{-2}\left(\sum_{j=1}^{n} h_{i}(s, t) \nu_{i}\right)^{2} \\
& +\Phi_{\theta}^{-2}\left\{\left(\sum_{i, j=1}^{n} a_{i j}(s, t) \nu_{i} \nu_{j}\right)^{2}+\left(\sum_{j=1}^{n} h_{i}(s, t) \nu_{i}\right)^{2}\right. \\
& \left.\quad \times \frac{\sum_{i=1}^{n}\left(h_{i}(s, t)^{2}+\left(\sum_{j=1}^{n} a_{i j}(s, t) \nu_{j}\right)^{2}\right)}{d^{2}}\right\} \\
& c_{2}(s, t: \theta)=2 \Phi_{\theta}^{-1}\left\{\left(\sum_{i, j=1}^{n} a_{i j}(s, t)^{2}+\sum_{i=1}^{n} h_{i}(s, t)^{2}\right) / d\right. \\
& \left.+\left|\sum_{i=1}^{n} h_{i}(s, t) \nu_{i}\right|\right\}+2 \\
& b(s, t: \theta)=\Phi_{\theta}^{-1} b(s, t)
\end{aligned}
$$

and we set 


$$
\begin{aligned}
\alpha_{\theta} & =\Phi_{\theta}^{-1}(\alpha-|v-n|) \\
d_{\theta} & =\Phi_{\theta}^{-1} d .
\end{aligned}
$$

It is necessary to hold

$$
\Phi_{\theta}^{-1} b(s, t) \leqslant \frac{d_{\theta}}{4} \min \left(1, \sqrt{\frac{\alpha_{\theta}(s, t)}{c_{2}(s, t: \theta)}}, \frac{\alpha_{\theta}(s, t)}{\sqrt{c_{1}(s, t: \theta)+4 \alpha_{\theta}^{2}}}\right)
$$

for all $\theta \in\left(0, \lambda_{\max }^{2} t\right)$. Indeed from the explicit forms of these quantities the existence of the smooth positive function $\gamma(s, t)$ is derived, in fact

$$
\inf _{\theta \in\left(0, \lambda_{\max }^{2} t_{0}^{2}\right)} \Phi_{\theta} \frac{d_{\theta}}{4} \min \left(1, \sqrt{\frac{\alpha_{\theta}}{c_{2}(\theta)}}, \frac{\alpha_{\theta}}{\sqrt{c_{1}(\theta)+4 \alpha_{\theta}^{2}}}\right)>0 .
$$

Then it suffices to show only

Lemma 5.1. (Local uniqueness near the boundary). Let $u(x, t)$ $\in C^{2}$ defined in a neighborhood of $\left(x_{0}, t_{0}\right)$ where $x_{0} \in S$. If $u(x, t)$ satisfies

$$
\begin{aligned}
& \int u\left(x, t_{0}\right)=\frac{\partial u}{\partial t}\left(x, t_{0}\right)=0 \quad \text { for } \quad x \in V \cap\left\{t=t_{0}, \Phi\right\}
\end{aligned}
$$

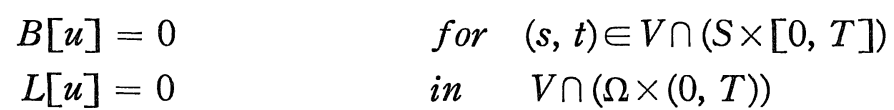

for some neighborhood $V$ of $\left(x_{0}, t_{0}\right)$, then $u(x, t)$ is identically zero in a neighborhood $V^{\prime}(\subset V)$ of $\left(x_{0}, t_{0}\right)$.

Proof. We can assume $\left(x_{0}, t_{0}\right)=(0,0)$. After the Folmgren transformation

$$
\begin{aligned}
& x^{\prime}=x \\
& t^{\prime}=|x|^{2}+t
\end{aligned}
$$

$\tilde{u}\left(x^{\prime}, t^{\prime}\right)=u(x, t)$

$$
\begin{array}{ll}
\tilde{L}[\hat{u}]=0 & \text { in } \quad F(V \cap(\Omega \times(0, T))) \\
\tilde{B}[\tilde{u}]=0 & \text { in } \quad F(V \cap(S \times[0, T]))
\end{array}
$$

where

$$
\begin{aligned}
\widetilde{L}= & \frac{\partial^{2}}{\partial t^{\prime 2}}+\Phi^{-1}(x, t)\left\{\sum_{i=1}^{n} 2\left(h_{i}-\sum_{j=1}^{n} a_{i j} x_{j}\right) \frac{\partial^{2}}{\partial t^{\prime} \partial x_{i}^{\prime}}\right. \\
& \left.-\sum_{i, j=1}^{n} a_{i j} \frac{\partial^{2}}{\partial x_{i}^{\prime} \partial x_{j}^{\prime}}\right\}+(\text { first order })
\end{aligned}
$$




$$
\begin{aligned}
& \tilde{B}=\frac{\partial}{\partial v_{t^{\prime}}}-\left(-\sum_{i=1}^{n} v_{i} x_{i}+\sigma_{1}\right) \frac{\partial}{\partial t^{\prime}}+\sigma_{2} \\
& \Phi=\left(1+\sum_{i=1}^{n} 2 h_{i} x_{i}-\sum_{i, j=1}^{n} a_{i j} x_{i} x_{j}\right) .
\end{aligned}
$$

By extending $\tilde{u}\left(x^{\prime}, t^{\prime}\right)$ by zero in the outside of $\left\{\left(x^{\prime}, t^{\prime}\right) ;\left|x^{\prime}\right|^{2} \geqslant t^{\prime}\right.$, $\left.t^{\prime} \geqslant 0\right\} \cap \Omega \times(0, T) \quad \tilde{u}\left(x^{\prime}, t^{\prime}\right) \in \mathcal{E}_{t}^{0}\left(H^{2}(\Omega)\right) \cap \mathcal{E}_{t}^{1}\left(H^{1}(\Omega)\right) \cap \mathcal{E}_{t}^{2}\left(L^{2}(\Omega)\right)$ and satisfies $\widetilde{L}[\tilde{u}]=0$ in $\Omega \times\left(0, k_{0}\right) \widetilde{B}[\tilde{u}]=0$ in $S \times\left[0, k_{0}\right]$ for some $k_{0}>0$ and $\tilde{u}\left(x^{\prime}, 0\right)=\frac{\partial \tilde{u}}{\partial t}\left(x^{\prime}, 0\right)=0$ in $\Omega$. Then $\tilde{L}, \widetilde{B}$ satisfy the condition (2.32) if $x$ is sufficiently small, therefore for some $k_{1}>0\left(k_{1} \leqslant k_{0}\right)$ the energy inequality (2.33) for $\widetilde{L}$ and $\widetilde{B}$ holds for $t \in\left(0, k_{1}\right)$. This shows that $\tilde{u}\left(x^{\prime}, t^{\prime}\right)$ is zero in $\Omega \times\left(0, k_{1}\right)$ from which it follows that $u(x, t)$ is zero in a neighborhood of $\left(x_{0}, t_{0}\right)$.

\section{References}

[1] Agmon, S., Problème mixtes pour les équations hyperboliques d'ordre supérieur, Colloques Internationaux du C. N. R. S. (1962), 13-18.

[2] Duff, G. F. D., A mixed problem for normal linear partial differential hyeprbolic equations of the second order, Canad. J. Math. 9 (1957), 141-160.

[3] Hersh, R., Mixed problem in several variables, J. Math. Mech. 12 (1963), 317-334.

[4] - On surface waves with finite and infinite speed of propagation, Arch. Rational Mech. Anal. 19 (1965), 308-316.

[5] Hörmander, L., Uniqueness theorems and estimates for normally hyperbolic partial differential equations of the second order, C. R. du douzième congrès des mathématiciens scandinaves (1953), 105-115.

[6] Ikawa, M., Mixed problems for hyperbolic equations of second order, J. Math. Soc. Japan 20 (1968), 580-608.

[7] - On the mixed problem for the wave equation with an oblique derivative boundary condition, Proc. Japan Acad. 44 (1968), 1033-1037.

[8] - A mixed problem for hyperbolic equations of second order with non-homogeneous Neumann type boundary condition, to appear in Osaka J. Math.

[9] — On the mixed problems for hyperbolic equations of second order, to appear in Suggaku. (Japanese)

[10] Krzyzanski M. and J. Schauder, Quasilineare Differentialgleichungen zweiter Ordnung vom hyperbolichen Typus, Gemischte Randwertaufgaben, Studia Math. 6 (1936), 162-189.

[11] Ladyzhenskaya, O. A., Mixed Problems for Hyperbolic Equations, Moscow, 1953 (Russian).

[12] - On the solution of non-stationary operator equations, Math. Sb. 39 (1956), 491-524 (Russian).

[13] Lee, K., A mixed problem for hyperbolic equations with time-dependent domain, J. Math. Anal. Appl. 16 (1966), 495-471.

[14] Lions, J. L., Equations Différentielles Opérationnelles, Springer, 1961.

[15] Mizohata, S., Theory of Partial Differential Equations, Iwanami, Tokyo, 1965 (Japanese). 
[16] Mizohata, S., Système hyperboliques, J. Math. Soc. Japan 11 (1959), 205-233.

[17] - Quelques problèmes au bord, du type mixte, pour des équations hyperboliques, Séminaire sur les équations aux dérivées partielles, Collège de France (1966-1967), 23-60.

[18] Miyatake, S., On some mixed problems for fourth order hyperbolic equations, J. Math. Kyoto Univ. 8 (1968), 285-311.

[19] Rogak, E. D., Mixed problem for the wave equation in a time dependent domain, Arch. Rational Meth. Anal. 22 (1966), 24-36. 
